\title{
Resonance ionization laser ion sources
}

\author{
U. Köster ${ }^{\mathrm{a}}$, \\ a CERN, CH-1211 Genève 23
}

\begin{abstract}
The three main requirements to the ion source of an ISOL facility are efficiency, selectivity and rapidity. For many metallic elements these requirements are ideally fulfilled by a resonance ionization laser ion source (RILIS). Presently such ion sources are used at the RIB facilities IRIS (Gatchina), ISOLDE (CERN), LISOL (Leuven), TIARA (Takasaki) and IMP (Lanzhou) to provide beams with low isobaric contamination. The isotopically pure beams enabled to make spectacular progress, for instance in nuclear spectroscopy of very rare isotopes. The scanning of the hyperfine structure with a small bandwidth laser allows moreover to separate individual isomers. The RILIS has also been used as a sensitive tool for atomic spectroscopy (measurement of the isotope shift and of nuclear moments) of exotic isotopes.
\end{abstract}

Key words: PACS: 29.25.Ni, 29.25.Rm, 32.10.Fn, 32.80.Fb

RNB-2000, ISOL, resonance ionization laser ion source, isomer separation, atomic spectroscopy

\section{Introduction}

Studies of exotic isotopes far from stability depend crucially on the availability of appropriate beams. To obtain a favorable signal to background ratio the experiments require a sufficient beam intensity and beam purity. A large number of radioactive isotopes can be provided by isotope separator on-line (ISOL) facilities. The main requirements to the ion source of an ISOL facility are efficiency, selectivity and rapidity. The latter reduces decay losses in the target and ion source system and directly translates into a higher release efficiency for short-lived nuclei.

Figure 1 shows different ways of ionization. Positive surface ionization (see left part) is very efficient for elements with an ionization potential smaller than

1 Email: Ulli.Koster@cern.ch

Article published in Elsevier Preprint NPA RNB5 proc. (2001) 1 


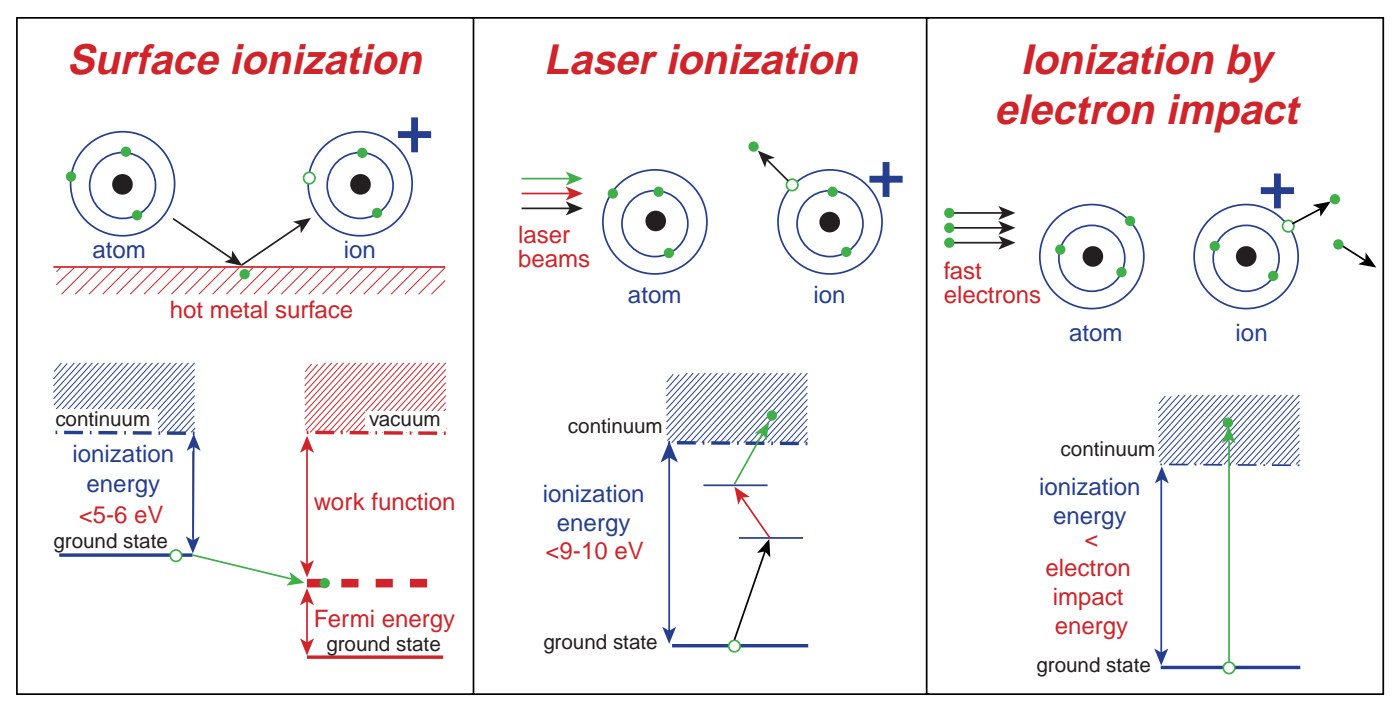

Fig. 1. Schematic representation of surface ionization, resonant photo ionization and ionization by electron impact.

the work function of the hot ionizer (noble metal surface). Thus alkalis can be ionized efficiently and selectively. The rare earths, the heavier alkaline earths and the heavier boron group elements are also surface ionized, though with reduced efficiency.

Ionization in plasma ion sources proceeds mainly by electron impact (see right part of figure 1) and atom-ion collisions. However, these processes are rather unselective. Practically every atom entering the plasma volume can get ionized regardless its chemical nature. Elemental selectivity can be achieved by thermodynamical (cold transfer line) or chemical (separation of molecules) "tricks" [1]. The first method is of advantage for low-melting elements with high vapor pressure (noble gases, $\mathrm{Cd}, \mathrm{Hg}$ ), the latter for reactive non-metals (C, N, O, Se, etc.) and metals which tend to form stable molecules (e.g. $\mathrm{SrF}^{+}$, $\mathrm{YF}^{2+}, \mathrm{BaF}^{+}$, lanthanide fluorides or oxides).

However, there is still a large number of elements which are neither well surface ionized, nor selectively separated with a plasma ion source. Also elements with high chemical affinity towards oxides (e.g. Be) are difficult to separate with a plasma ion source. Most plasma ion sources contain insulators with oxide surfaces. The latter can "trap" the radio-isotopes and cause huge decay losses. Thus, for many metals with ionization potentials between 6 and $10 \mathrm{eV}$ and low vapor pressure at temperatures below $2000{ }^{\circ} \mathrm{C}$ there remains a "gap" between surface ion source and plasma ion source. This can be filled with the method of resonant photo ionization. 


\section{Resonance Ionization Laser Ion Sources}

In a resonance ionization laser ion source RILIS $^{2}$ ) the valence electron is excited by resonant photon absorption via several intermediate steps into the continuum, see middle part of figure 1. The position of the excited levels is specific for each element. Tuning the laser wavelengths to this "fingerprint" provides an ionization method with high intrinsic elemental selectivity.

Resonance ionization was already applied for nearly every element, see e.g. $[3,4]$ for an overview of the used schemes. However, for transitions to high-lying $(>6 \mathrm{eV})$ first excited states of non-metals, laser beams in the far ultraviolet $(\lambda<200 \mathrm{~nm})$ are required. These can only be produced with low intensity and/or low duty cycle. Thus, these elements are less suitable for efficient laser ionization. Alternative separation methods for non-metals have been discussed above.

To achieve maximum ionization efficiency, intense laser beams are used for excitation and ionization. The laser beams need to have a sufficient overlap in time and space with the "atomic beam" of radio-isotopes. Since presently only pulsed lasers can provide sufficient beam power, the atoms have to be confined in the interaction region for a while. Different methods can be used for such a "storage" of the atoms.

\subsection{Hot cavity RILIS}

The atoms can be "stored" in a hot cavity with a small outlet hole [5]. The average residence time of an atom in the used hot cavities is of the order of some $0.1 \mathrm{~ms}$. Thus a laser system with a repetition rate of the order of $10 \mathrm{kHz}$ is required to assure that each atom has at least one chance to interact with the laser beams. Presently at this repetition rate mainly ${ }^{3}$ copper vapor lasers (CVL) are used to provide several $10 \mathrm{~W}$ beam power at 511 and $578 \mathrm{~nm}$ to pump the tunable dye lasers. Via frequency doubling and tripling in nonlinear crystals wavelengths in the range of about 210 to $1000 \mathrm{~nm}$ can be provided. However, the available beam power drops significantly towards the UV region and only strong transitions can be saturated. Weaker transitions or direct transitions to the continuum remain often unsaturated and limit the ionization efficiency. The ionizer cavity is in its simplest version identical to

$\overline{2}$ In the following, this name will be used to differentiate from laser ion sources which produce unselectively multi-charged ions in a plasma created by a highintensity laser pulse, see e.g. ref. [2] for the latter.

3 At the TIARA facility Nd-YAG and excimer lasers with a maximum repetition rate of $400 \mathrm{~Hz}$ are used. 
the ionizer tube of a surface ion source. A Nb, Ta or W tube can be heated to well above $2000{ }^{\circ} \mathrm{C}$. Thus the delay in the target and ion source system is normally dominated by the target. Due to the high temperature of the ionizer cavity, most molecules are dissociated and the laser ionized beam consists purely of atomic ions.

Unfortunately the hot cavity acts simultaneously as a surface ionizer for elements with low ionization potential. This, sometimes strong, isobaric background has to be suppressed by additional means:

- Ionizer cavities from low work function material (e.g. TaC) can reduce such background while maintaining a high laser ionization efficiency $[5,6]$. However, not all materials are suitable for on-line use where the surface properties of the ionizer cavities need to remain stable for a relatively long time (several days). A reduced cavity temperature will reduce the surface ionized background, but will also increase the delay in the cavity. Thus, for shortlived isotopes a compromise has to be found between background suppression and decay loss minimization.

- A fast beam gate can be opened for some ten $\mu$ s to let pass only the short bunches of laser ionized ions while cutting off a major part of the dc released surface ionized background [5]. Very thin ionizer cavities (e.g. a Nb tube with $60 \mu \mathrm{m}$ walls) with a high longitudinal field of some $\mathrm{V} / \mathrm{cm}$ provide an optimum bunching and allow to increase the selectivity via this "microgating technique" by up to one order of magnitude $[7,8]$.

- Due to their higher ionization potential, the nuclides to be laser ionized diffuse mainly as atoms from the target to the ionizer cavity, while the surface ionizable isobars are partially ionized already inside the target. A potential gradient applied along target or transfer line allows to push the disturbing isobars back into the target. This effect delays their release from the target and ion source system. A real background suppression can be obtained by extracting the surface ionized ions through a hole in the "backside" of the target or by using an ac heating current for a bunched release of the surface ionized background [9].

Hot cavity RILIS are presently under operation at ISOLDE (CERN), IRIS (Gatchina), TIARA (Takasaki) and IMP (Lanzhou). Moreover, radioactive isotopes are studied off-line with similar systems in Mainz (see e.g. ref. $[10,11]$ ).

It has to be kept in mind that, before being ionized, the radio-isotopes have to be released from the target. Thus refractory elements could well be laser ionized when supplied as atomic beam, but they are practically not released from a thick ISOL target. To avoid such an undesired chemical selectivity of the target, it is better to use a buffer gas cell RILIS for refractory elements. 


\subsection{Buffer gas cell RILIS}

In a buffer gas cell the atoms can be stored for a longer time (some tens ms) which gives less stringent requirements to the repetition rate of the pumping lasers. Thus e.g. excimer lasers with some $100 \mathrm{~Hz}$ repetition rate can be used. With a $\mathrm{XeCl}$ excimer beam of $308 \mathrm{~nm}$ the dye lasers can be pumped directly down to about $320 \mathrm{~nm}$. The high pulse power simplifies efficient frequency doubling and for many elements all transitions can be saturated. However, losses occur between ionization and ion extraction. Formation of molecular ions can disperse the laser ionized isotopes over several sidebands, thus reducing the efficiency of atomic ion separation. See [12-14] for a discussion of these loss processes in great detail.

With all transitions being saturated, the efficiency of a buffer gas cell RILIS is less dependent on the element than on the used production reaction which determines the capture efficiency. At LISOL efficiencies of $5 \%$ for light-ion induced fusion reactions, $0.2 \%$ for proton-induced fission of ${ }^{238} \mathrm{U}$ and $0.05 \%$ for heavy-ion induced fusion reactions were measured [15]. The main background in a buffer gas cell RILIS comes from remaining ions produced by the primary beam impact which were not neutralized or removed. Thus the selectivity is typically of the order of 100 .

A variation of the buffer gas cell RILIS with very fast extraction $(\approx 1 \mathrm{~ms})$ is used for atomic spectroscopy at the MPI for Nuclear Physics in Heidelberg [16].

\subsection{Pulsed desorption RILIS}

A third way of confinement is the continuous adsorption of the atoms onto a "cold" surface. Shortly before firing the ionizing lasers, the surface is heated rapidly (e.g. by pulsed laser desorption with an intense laser beam) and the atoms get desorbed. The efficiency depends strongly on the spatial and temporal overlap between ionizing laser beams and desorbed atom cloud. For not too short-lived radio-isotopes repetition rates of some 1-50 Hz are sufficient, which can be provided by relatively cheap Nd-YAG lasers. This method has been applied for resonance ionization spectroscopy experiments (see e.g. [17,18]) and was proposed as ion source at an ISOL facility $[19,20]$.

Table 1 shows the used ionization schemes and measured efficiencies of elements which were ionized at different RILIS facilities. Other elements for which the ionization schemes were tested off-line are summarized in figure 2. 
Table 1

Ionized elements and produced radio-isotopes at different RILIS facilities. The quoted references give details of the set-up and some on-line applications of the ionized elements. Most given efficiencies were measured off-line. For the LISOL efficiencies see text.

\begin{tabular}{|c|c|c|c|c|c|c|c|}
\hline $\begin{array}{l}\text { Ele- } \\
\text { ment }\end{array}$ & $\begin{array}{c}\mathrm{IP} \\
(\mathrm{eV})\end{array}$ & $\begin{array}{c}\lambda_{1} \\
\mathrm{~nm}\end{array}$ & $\begin{array}{c}\lambda_{2} \\
\mathrm{~nm}\end{array}$ & $\begin{array}{c}\lambda_{3} \\
\mathrm{~nm}\end{array}$ & $\begin{array}{l}\text { Eff. } \\
(\%)\end{array}$ & $\begin{array}{c}\text { Separated } \\
\text { isotopes }\end{array}$ & Ref. \\
\hline \multicolumn{7}{|c|}{ ISOLDE, CERN } & {$[5,21]$} \\
\hline $\mathrm{Li}$ & 5.39 & 670.8 & 610.4 & $610.4,670.8$ & & & {$[5]$} \\
\hline $\mathrm{Be}$ & 9.32 & 234.9 & 297.3 & - & $>7$ & $7,9-12,14$ & {$[22]$} \\
\hline $\mathrm{Mg}$ & 7.65 & 285.2 & 552.8 & 578 & 10 & Stable & {$[21]$} \\
\hline $\mathrm{Al}$ & 5.99 & $308.2,309.3$ & 511,578 & - & $>20$ & Stable & \\
\hline $\mathrm{Ca}$ & 6.11 & 272.2 & 511,578 & - & 0.45 & Stable & \\
\hline $\mathrm{Mn}$ & 7.43 & 279.8 & 628.3 & 511 & 19 & $48-69$ & {$[23,24]$} \\
\hline $\mathrm{Co}$ & 7.88 & 304.4 & 544.5 & 511,578 & $>4$ & Stable & \\
\hline $\mathrm{Ni}$ & 7.64 & 305.1 & 611.1 & 748.2 & $>6$ & $56-70$ & {$[25]$} \\
\hline $\mathrm{Cu}$ & 7.73 & 327.4 & 287.9 & - & $>7$ & $57-78$ & {$[26]$} \\
\hline $\mathrm{Zn}$ & 9.39 & 213.9 & 636.2 & 511 & 5 & $58-73$ & {$[27]$} \\
\hline Ga & 6.00 & 287.4 & 511,578 & - & 21 & $70-85$ & \\
\hline $\mathrm{Ag}$ & 7.58 & 328.1 & 546.6 & 511 & 14 & $101-129$ & {$[7]$} \\
\hline $\mathrm{Cd}$ & 8.99 & 228.8 & 643.8 & 511 & 10 & $98-132$ & [28] \\
\hline In & 5.79 & 303.9 & 511,578 & - & & $100-112$ & \\
\hline $\mathrm{Sn}$ & 7.34 & 300.9 & 811.4 & 823.5 & $\approx 10$ & $105-137$ & {$[29]$} \\
\hline $\mathrm{Tm}$ & 6.18 & 589.6 & 571.2 & 575.5 & & Stable & {$[5]$} \\
\hline $\mathrm{Yb}$ & 6.25 & 555.6 & 581.1 & 581.1 & 15 & $157-167$ & {$[5]$} \\
\hline $\mathrm{Tl}$ & 6.11 & 276.8 & 511,578 & - & 27 & Stable & \\
\hline $\mathrm{Pb}$ & 7.42 & 283.3 & 600.2 & 511,578 & 3 & $184-215$ & {$[30]$} \\
\hline $\mathrm{Bi}$ & 7.29 & 306.8 & 555.2 & 511,578 & 6 & Stable & \\
\hline \multicolumn{7}{|c|}{ TIARA, Takahashi } & {$[31]$} \\
\hline $\mathrm{Na}$ & 5.14 & 589.8 & 568.4 & 532 & & Stable & {$[32]$} \\
\hline $\mathrm{Ba}$ & 5.21 & 350.1 & 532 & & 0.04 & 127 & {$[31]$} \\
\hline $\mathrm{La}$ & 5.58 & 593.0 & 820.5 & 532 & & Stable & {$[31]$} \\
\hline \multicolumn{7}{|c|}{ IRIS, Gatchina } & {$[33,34]$} \\
\hline $\mathrm{Nd}$ & 5.53 & 588.8 & 596.9 & 596.9 & 20 & Stable & {$[35]$} \\
\hline Но & 6.02 & 592.1 & 572.5 & 626.8 & 5 & 152 & {$[35]$} \\
\hline $\mathrm{Tm}$ & 6.18 & 597.1 & 600.3 & 552.4 & $10-15$ & $153-169$ & {$[9,35]$} \\
\hline $\mathrm{Yb}$ & 6.25 & 555.6 & 581.1 & 581.1 & 30 & $154-176$ & {$[9,35]$} \\
\hline \multicolumn{7}{|c|}{ IMP, Lanzhou } & {$[36]$} \\
\hline $\mathrm{Tm}$ & 6.18 & 589.6 & 571.2 & 575.5 & & Stable & {$[36]$} \\
\hline $\mathrm{Yb}$ & 6.25 & 555.6 & 581.1 & 581.1 & 0.1 & 167 & {$[36]$} \\
\hline \multicolumn{7}{|c|}{$\begin{array}{ll}\text { LISOL, Leuven } \\
\end{array}$} & {$[37]$} \\
\hline $\mathrm{Ti}$ & 6.82 & $337.8,395.8$ & $384.1,339.4$ & - & $*$ & 42,43 & {$[14]$} \\
\hline Co & 7.86 & 230.9 & 481.9 & - & $*$ & $54,55,65-70$ & {$[38]$} \\
\hline $\mathrm{Ni}$ & 7.64 & 232.0 & 537.8 & - & $*$ & $54,55,68-74$ & {$[39]$} \\
\hline $\mathrm{Cu}$ & 7.73 & 244.2 & 441.6 & - & $*$ & $70-77$ & {$[40]$} \\
\hline $\mathrm{Ru}$ & 7.36 & 228.5 & 553.1 & - & $*$ & 89-91 & {$[41]$} \\
\hline $\mathrm{Rh}$ & 7.46 & 232.3 & 572.6 & - & * & $90-95,98,112-114$ & [41] \\
\hline
\end{tabular}




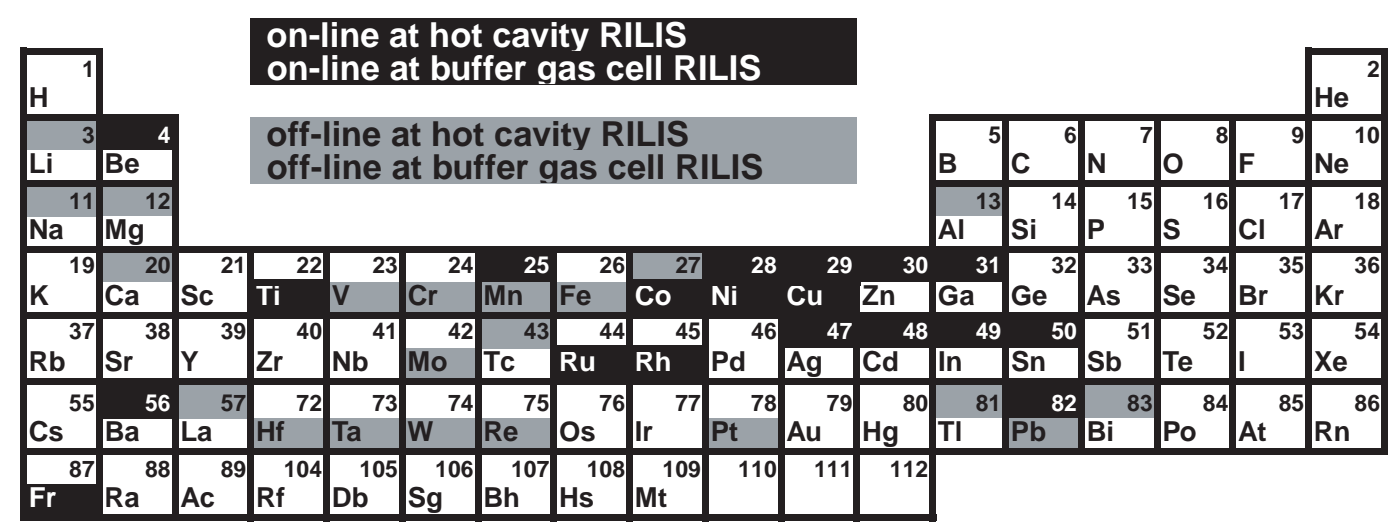

\begin{tabular}{|c|c|c|c|c|r|r|r|r|r|r|r|r|r|r|}
\hline $\mathrm{Ce}^{58}$ & $\mathrm{Pr}^{59}$ & $\mathrm{Nd}^{60}$ & $\mathrm{Pm}^{61}$ & $\mathrm{Sm}^{62}$ & $\mathrm{Eu}^{63}$ & $\mathrm{Gd}^{64}$ & $\mathrm{~Tb}^{65}$ & $\mathrm{Dy}^{66}$ & $\mathrm{Ho}^{67}$ & $\mathrm{Er}^{68}$ & $\mathrm{Tm}^{69}$ & $\mathrm{Yb}^{70}$ & $\mathrm{Lu}^{71}$ \\
\hline $\mathrm{Th}^{90}$ & $\mathrm{~Pa}^{91}$ & $\mathrm{U}^{92}$ & $\mathrm{~Np}^{93}$ & $\mathrm{Pu}^{94}$ & $\mathrm{Am}^{95}$ & $\mathrm{Cm}^{96}$ & $\mathrm{Bk}^{97}$ & $\mathrm{Cf}^{98}$ & $\mathrm{Es}^{99}$ & $\mathrm{Fm}^{100}$ & $\mathrm{Md}^{101}$ & $\mathrm{No}^{102}$ & $\mathrm{Lr}^{103}$ \\
\hline
\end{tabular}

Fig. 2. Elements ionized on- or off-line in hot cavity RILIS (top of squares) and buffer gas cell RILIS (bottom of squares).

The complementarity of the hot cavity RILIS and the buffer gas cell RILIS is nicely seen for elements in the vicinity of nickel: manganese is rapidly released from a standard ISOLDE uranium carbide/graphite target and isotopes up to ${ }^{69} \mathrm{Mn}$ could be separated [23]. Nickel (and presumably also the chemically similar elements iron and cobalt) are very slowly released from the present ISOLDE targets [25]. Thus only longer-lived nuclides (up to ${ }^{70} \mathrm{Ni}$ ) are separated with reasonable efficiency. More short-lived nickel isotopes are better produced at LISOL. Presently neutron-rich isotopes up to ${ }^{74} \mathrm{Ni}$ [39] and ${ }^{70} \mathrm{Co}$ [42] are available there. For copper the release at ISOLDE is again sufficiently fast and the beam intensities [4] outnumber those of LISOL [40] for all isotopes. Figure 3 shows a comparison of gamma ray spectra measured in the beta-decay of mass 68 nuclei at LISOL and ISOLDE.

\section{$3 \quad$ Atomic physics effects}

Using an atomic physics technique for the ionization, it is evident that one has to take care of some specific atomic physics effects.

\subsection{Fine structure}

Many elements show a split ground state or other low-lying states. The thermal distribution in a gas cell or a hot cavity spreads the population over several of these states. Thus, only a fraction of the atoms can be excited in the first step and the laser ionization efficiency is reduced correspondingly. 

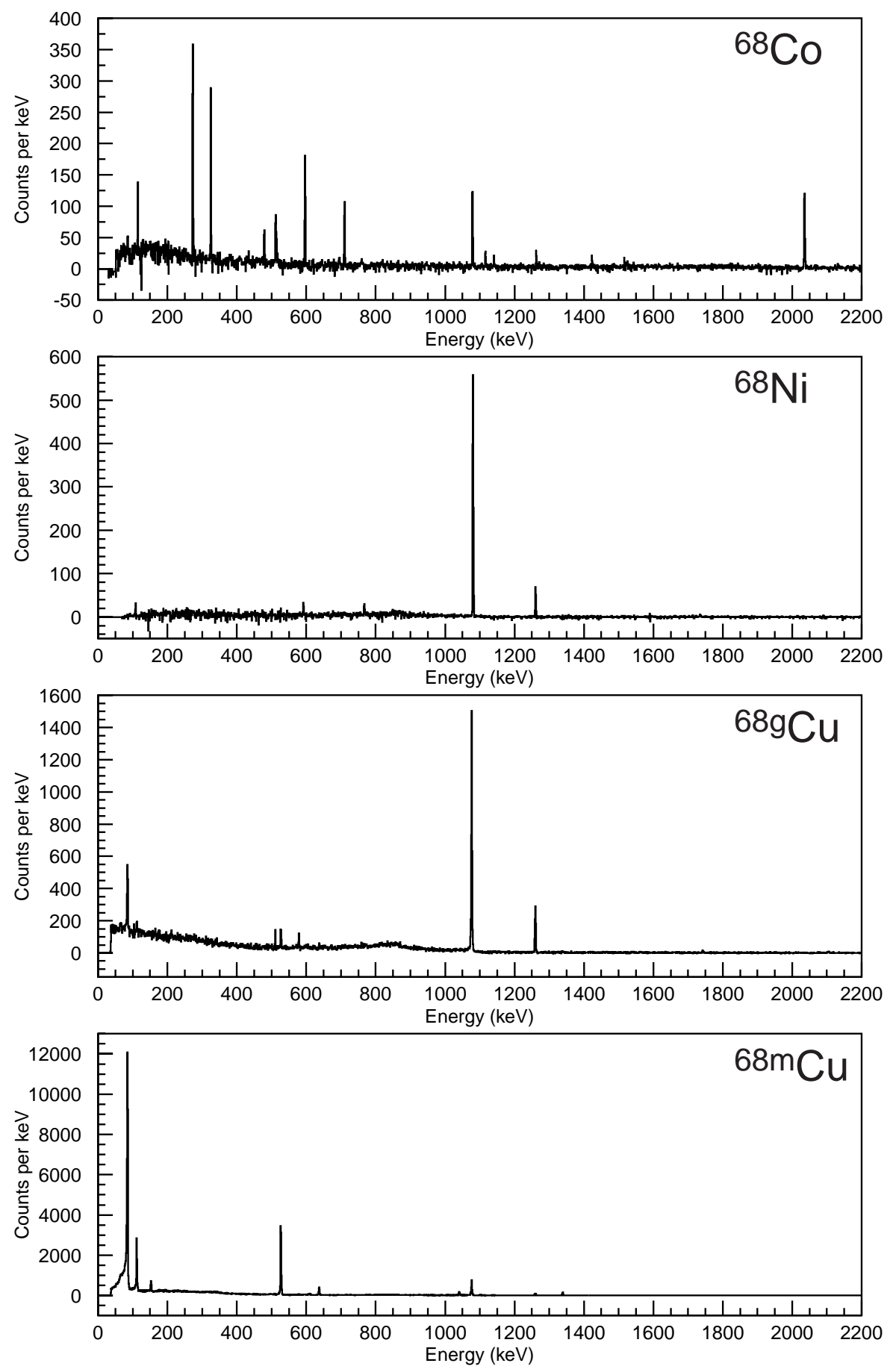

Fig. 3. Gamma ray spectra measured for the beta-decay of ${ }^{68} \mathrm{Co}$ (actually a mixture of ${ }^{68 g} \mathrm{Co}$ and ${ }^{68 m} \mathrm{Co}$ ) [42] and ${ }^{68} \mathrm{Ni}$ [43] at LISOL and for ${ }^{68 g} \mathrm{Cu}$ and ${ }^{68 m} \mathrm{Cu}$ measured at ISOLDE $[44,45]$. See section 3.3 for the separation of the two isomers. The shown spectra were taken with the frequency of the first excitation step at $30535.66 \mathrm{~cm}^{-1}$ $\left({ }^{68 g} \mathrm{Cu}\right)$ and $30535.36 \mathrm{~cm}^{-1}\left({ }^{68 m} \mathrm{Cu}\right)$ respectively, compare figure 4 . The beta-decay of ${ }^{68} \mathrm{Ni}$ proceeds predominantly to the $1^{+}$ground state of ${ }^{68} \mathrm{Cu}$, thus mainly gamma rays from the daughter decay are observed. All spectra are background substracted (i.e. "laser-on" minus "laser-off"). 
This problem occurs e.g. for Al, Ti, Co, Ni, In, Sn, etc. The efficiency can be increased by simultaneous excitation from several occupied levels. This was already successfully applied for the ionization of aluminum $\left(\lambda_{1}=308.2\right.$ and $309.3 \mathrm{~nm}$ ) at the ISOLDE RILIS, but more complicated ionization schemes (e.g. Ni or $\mathrm{Sn}$ ) would require the installation of additional dye lasers.

\subsection{Isotope shift}

The atomic levels of different isotopes of the same element have not exactly equal energy, but show a characteristic difference, the isotope shift. The isotope shift can exceed the Doppler width of the resonance line considerably for light nuclei $(A \lesssim 30)$ and for many lanthanides and heavy elements $(Z \gtrsim 80)$. To reach an optimum ionization efficiency the laser wavelength has to be retuned when changing from one isotope to another. While this makes the RILIS operation more tedious, on the other hand it allows to use the RILIS as a very sensitive tool for atomic spectroscopy applications, see e.g. ref. [9].

\subsection{Hyperfine splitting}

The interaction of the electrons with the angular momentum of the nucleus leads to a splitting of the atomic energy levels into a number of hyperfine components. With sufficient resolution these can be observed as individual peaks in a scan of the respective transition frequency. If the hyperfine splitting of two isomers differs significantly, a tuning of the laser wavelength allows to ionize preferentially one of the isomers, i.e. to perform an isomer separation. This was applied on-line for samarium and thulium [46], silver [47] and copper isomers [45]. Figure 4 shows the separation obtained in the case of ${ }^{68 g, m} \mathrm{Cu}$.

Moreover, with known nuclear and electronic spin, an accurate scan of the hyperfine splitting can be used to determine directly nuclear moments [26]. Although still far less accurate than e.g. a measurement via nuclear magnetic resonance (NMR) this method is very efficient and allows to reach far out to exotic nuclei.

For a hot cavity RILIS the resolution of atomic spectroscopy is limited by the Doppler broadening. A Doppler-free two photon spectroscopy could thus enhance the resolution considerably [48]. In a buffer gas cell RILIS the resonance width is mainly determined by the homogeneous pressure broadening [37]. Thus, a high gas pressure (which is necessary for a good stopping efficiency) makes this kind of RILIS less suitable for spectroscopic applications. 


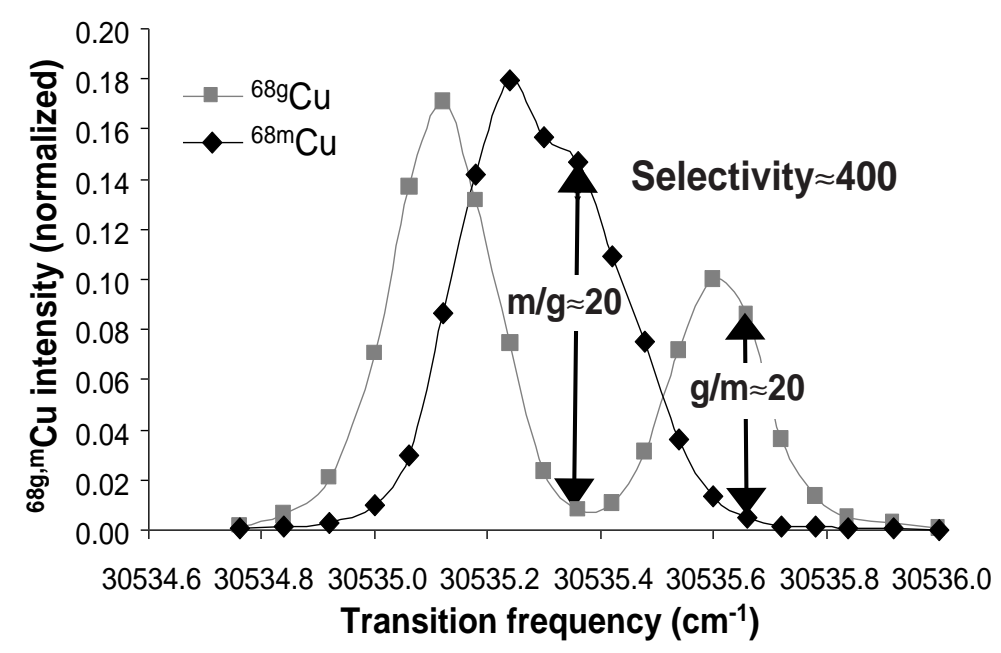

Fig. 4. Isomer separation of ${ }^{68 g} \mathrm{Cu}$ and ${ }^{68 m} \mathrm{Cu}$. Note that it would be extremely difficult to obtain a similar separation factor with a conventional mass separator, requiring a mass resolving power $>10^{5}$ in this case.

\subsection{Nuclear polarized beams}

By absorption of circularly polarized light the polarization is transferred to the excited state of the atom. The coupling of electronic and nuclear spin can transfer this polarization to the nucleus. Thus laser ionized beams with a certain degree of nuclear polarization (e.g. some 10 to $20 \%$ for alkalis [49]) could be produced. A still higher polarization could be obtained by additional optical pumping in the ionizer cavity prior to ionization. The practical implementation of this method (beam transport without polarization loss, etc.) is presently under preparation at ISOLDE.

\section{Summary and outlook}

In principle resonant photo ionization may be applied for all metallic elements. The optimum choice of the RILIS type (hot cavity or buffer gas cell) depends less on the RILIS itself than on the release characteristics of the associated target.

The ionization efficiencies of a RILIS are still lower than those of a plasma ion source which might reach $50 \%$ or more. However, it has to be kept in mind that e.g. in nuclear spectroscopy the reduced intensity is often more than compensated by an increased beam purity. An additional coincidence requirement which might be necessary to extract useful information from a mixture of different nuclei can easily cost one order of magnitude in the number of detected events. Moreover the possibility to "switch" a certain isotope with 
the laser beam "on" and "off" gives a unique possibility for the identification of new nuclei.

There is strong economic interest to make uranium enrichment by resonant laser ionization competitive to the "classical" methods (diffusion, centrifugation, etc.). Important R\&D for such a "large-scale RILIS" was already performed in France (SILVA project [50]) and the U.S. (AVLIS project [51]). The RILIS application at ISOL facilities could profit from the effort which is spent on this development of efficient and reliable laser systems matching exactly the requirements of a hot cavity type RILIS.

\section{Acknowledgments}

I am very grateful for numerous contributions to this article from members of the RILIS groups at ISOLDE, LISOL, IRIS, IMP and TIARA.

\section{References}

[1] U. Köster for the ISOLDE Collaboration, Radiochim. Acta 89 (2001) in press.

[2] P. Fournier et al., Rev. Sci. Instr. 71 (2000) 1405-1408.

[3] V.S. Letokhov, Laser photoionization spectroscopy, Academic Press, Orlando (1987).

[4] Ulli Köster, Yields and spectroscopy of radioactive isotopes at LOHENGRIN and ISOLDE, Ph.D. thesis, TU München (2000).

[5] V.I. Mishin et al., Nucl. Instr. Meth. B 73 (1993) 550-560.

[6] V.I. Beznosjuck, D.V. Fedorov, S.Yu. Orlov, M.D. Seliverstov and V.I. Tikhonov, Nucl. Instr. Meth. B 126 (1997) 92-94.

[7] Y. Jading et al., Nucl. Instr. Meth. B 126 (1997) 76-80.

[8] J. Lettry et al., Rev. Sci. Instr. 69 (1998) 761-763.

[9] A.E. Barzakh et al., Phys. Rev. C 61 (2000) 034304.

[10] W. Ruster et al., Nucl. Instr. Meth. A 281 (1989) 547-558.

[11] N. Erdmann et al., J. Alloys and Compounds 271-273 (1998) 837-840.

[12] Y. Kudryavtsev et al., Rev. Sci. Instr. 69 (1998) 738-740.

[13] M. Huyse, Nucl. Phys. A (2001) Proc. of RNB5. 
[14] Yu. Kudryavtsev et al., Nucl. Instr. Meth. B (2001) in press.

[15] Yu. Kudryavtsev et al., Nucl. Phys. A (2001) Proc. of RNB5.

[16] H. Backe et al., Phys. Rev. Lett. 80 (1998) 920-923.

[17] U. Krönert et al., Nucl. Instr. Meth. A 300 (1991) 522-537.

[18] F. Le Blanc et al., Phys. Rev. Lett. 79 (1997) 2213-2216.

[19] W.M. Fairbank and H.K. Carter, Nucl. Instr. Meth. B 26 (1987) 357-361.

[20] V. Sebastian, T. Budiman and G. Huber, Nucl. Instr. Meth. B 126 (1997) $73-75$.

[21] V.N. Fedoseyev, in Applications of Accelerators in Research and Industry, Proc. of the Fifteenth Int. Conf., Denton, edited by J.L. Duggan and I.L. Morgan, AIP Conf. Proc. 475, Woodbury, NY (1998) pp. 296-300.

[22] U. Köster et al., in Exotic Nuclei and Atomic Masses, ENAM'98, Bellaire, Michigan, June 1998, edited by B.M. Sherill, D.J. Morrissey and C.N. Davids, AIP Conf. Proc. 455, Woodbury, NY (1998) pp. 989-994.

[23] M. Hannawald et al., Phys. Rev. Lett. 82 (1999) 1391-1394.

[24] M. Oinonen et al., Hyp. Int. 127 (2000) 431-436.

[25] A. Jokinen et al., Nucl. Instr. Meth. B 126 (1997) 95-99.

[26] U. Köster et al., Nucl. Instr. Meth. B 160 (2000) 528-535.

[27] A. Jokinen et al., Eur. Phys. J. A 3 (1998) 271-276.

[28] M. Hannawald et al., Phys. Rev. C 62 (2000) 054301.

[29] V.N. Fedoseyev et al., Hyp. Int. 127 (2000) 409-416.

[30] H. De Witte et al., in 3rd Conf. on Exotic Nuclei and Atomic Masses, July 2001, Hämeenlinna, Finland (submitted).

[31] M. Koizumi, A. Osa, T. Sekine and M. Kubota, Nucl. Instr. Meth. B 126 (1997) 100-104.

[32] Mitsuo Koizumi, JAERI Takasaki, priv. comm.

[33] G.D. Alkhazov et al., Nucl. Instr. Meth. A 306 (1991) 400-402.

[34] A.E. Barzakh, V.P. Denisov, D.V. Fedorov, S.Yu. Orlov and M.D. Seliverstov, Nucl. Instr. Meth. B 126 (1997) 85-87.

[35] Vladimir N. Panteleyev, PNPI Gatchina, priv. comm.

[36] Zh. Zhizheng et al., Chin. J. Nucl. Phys. 19 (1997) 48-52.

[37] Y. Kudryavtsev et al., Nucl. Instr. Meth. B 114 (1996) 350-365.

[38] W.F. Mueller et al., Phys. Rev. Lett. 83 (1999) 3613-3616. 
[39] S. Franchoo et al., Phys. Rev. Lett. 81 (1998) 3100-3103.

[40] K. Kruglov et al., Nucl. Phys. A (2001) Proc. of RNB5.

[41] M. Górska et al., in Pingst 2000, Intern. workshop on selected topics on $N=Z$ nuclei, Lund, edited by D. Rudolph and M. Hellström, Div. of Cosmic and Subatomic Physics, Lund University (2000) pp. 108-112.

[42] W.F. Mueller et al., Phys. Rev. C 61 (2000) 054308.

[43] Serge Franchoo, Evolution of nuclear structure towards ${ }^{78} \mathrm{Ni}$ investigated by the $\beta$ decay of laser-ionized ${ }^{68-74} \mathrm{Ni}$, Ph.D. thesis, K.U. Leuven (1999).

[44] Jan Van Roosbroeck, K.U. Leuven, priv. comm.

[45] U. Köster et al., Hyp. Int. 127 (2000) 417-420.

[46] V.I. Mishin et al., Opt. Comm. 61 (1987) 383-386.

[47] V. Sebastian et al., in Exotic Nuclei and Atomic Masses, ENAM'98, Bellaire, Michigan, June 1998, edited by B.M. Sherill, D.J. Morrissey and C.N. Davids, AIP Conf. Proc. 455, Woodbury, NY (1998) pp. 126-129.

[48] Viatcheslav I. Mishin, Inst. of Spectroscopy, Russian Academy of Sciences, Troitsk, priv. comm.

[49] G. Neyens, G. S'heeren and R. Coussement, Nucl. Instr. Meth. B 122 (1997) $121-126$.

[50] J. Cazalet, Revue Générale Nucléaire 2 (Mars-Avril 1996) 20-23.

[51] S. Hargrove, Science and Technology Review, Lawrence Livermore National Laboratory (May 2000) 13-21. 